Artículo

\title{
La biofortificación con selenio mejora los compuestos bioactivos y la actividad antioxidante en chile jalapeño
}

\author{
Jazmín Monserrat Gaucin-Delgado ${ }^{1}$ \\ Pablo Preciado-Rangel ${ }^{1 \S}$ \\ Uriel González-Salas ${ }^{2}$ \\ Ernesto Sifuentes-Ibarra ${ }^{3}$ \\ Fidel Núñez-Ramírez ${ }^{4}$ \\ Jorge Arnaldo Orozco Vidal ${ }^{1}$
}

${ }^{1}$ Posgrado en Agua y Suelo-Tecnológico Nacional de México-Instituto Tecnológico de Torreón. Carretera Torreón-San Pedro km 7.5, Torreón, Coahuila, México. CP. 27170. (jazmontse@hotmail.com; joorvi66@ hotmail.com). ${ }^{2}$ Facultad de Agricultura y Zootecnia-Universidad Juárez del Estado de Durango. Carretera Gómez Palacio-Tlahualilo km 32, Venecia, Gómez Palacio, Durango, México. CP. 35000 (u.gonzalez@ujed.mx). ${ }^{3}$ Campo Experimental Valle del Fuerte-INIFAP. Carretera internacional MéxicoNogales km 1609, Juan José Ríos, Sinaloa, México. CP. 81110. (eblnat68@ gmail.com). ${ }^{4}$ Instituto de Ciencias Agrícolas de la Universidad Autónoma de Baja California. Carretera a Delta s/n, ejido Nuevo León, Mexicali, Baja California, México. CP. 21705. (fidel.nunez@uabc.edu.mx).

${ }^{\S}$ Autor para correspondencia: ppreciador@yahoo.com.mx.

\section{Resumen}

El selenio (Se) es un oligoelemento esencial para la salud humana y en las plantas es considerado un elemento benéfico, al ser un promotor del crecimiento y un detonador de la respuesta antioxidante en las plantas. La biofortificación con Se, tiene como objetivo obtener alimentos ricos en este oligoelemento, de alta calidad nutricional que ayuden a combatir los problemas de desnutrición en la población. El presente trabajo tiene como objetivo evaluar la capacidad del selenato $\left(\mathrm{Na}_{2} \mathrm{SeO}_{4}\right)$ sobre el rendimiento, biosíntesis de compuestos bioactivos y su acumulación en frutos de chile. Para ello cinco tratamientos fueron aplicados vía solución nutritiva: 0, 1.5, 3, 4.5 y $6 \mathrm{mg} \mathrm{L}^{-1}$. En la cosecha, se cuantificó la calidad nutracéutica, la acumulación de Se en frutos, así como el rendimiento del cultivo. La biofortificación con Se modificó positivamente la biosíntesis de compuestos bioactivos y su concentración en fruto, sin disminución en el rendimiento. La incorporación de Se en la solución nutritiva es una opción para obtener alimentos funcionales con una calidad nutracéutica y con la posibilidad de mejorar la salud pública tras su consumo.

Palabras clave: Capsicum annuum L., alimento funcional, compuestos bioactivos.

Recibido: junio de 2021

Aceptado: septiembre de 2021 


\section{Introducción}

El selenio (Se) es un oligoelemento importante en la nutrición humana, es esencial para formar proteínas y cofactor de enzimas antioxidantes como el glutatión peroxidasa (GPX), que protege el cuerpo humano al catalizar la reducción de las especies reactivas de oxígeno (ERO) (Schiavon et al., 2020). Además, en los mamíferos, el Se forma al menos 25 selenoproteínas que cumplen funciones antioxidantes, catalíticas, antiinflamatorias, antivirales y antitumorales (Avery y Hoffmann, 2018).

Una deficiencia de este elemento provoca problemas de salud, incluida la enfermedad de Keshan (una miocardiopatía endémica), enfermedad de Kashin-Beck (osteoartropatía deformante endémica) Ulloa et al. (2021), aceleración de procesos cancerígenos en la próstata (Sonkusre, 2020), problemas de fertilidad y debilitamiento del sistema inmunológico sistema de defensa contra enfermedades infecciosas virales como la influenza, virus de la inmunodeficiencia humana, distrofia muscular y fibrosis quística (Khurana et al., 2019). De acuerdo a la Organización Mundial de la Salud, el consumo de Se en la dieta humana debe fluctuar entre 55 y $200 \mu \mathrm{g} \mathrm{día}^{-1}$ en adultos (Górska et al., 2021). La forma más común por la cual el organismo humano adquiere el Se es a través del consumo de alimentos, tales como la carne o el pescado (Sariñana-Navarrete et al., 2021), que aportan un alto porcentaje de Se a la ingesta diaria requerida.

En el mundo, existen alrededor de mil millones de personas con deficiencias de Se, debido principalmente al consumo de dietas basadas en vegetales Błażewicz et al. (2020), las cuales contienen bajas concentraciones de Se, ya que este elemento se encuentra en pequeñas cantidades en el suelo (López et al., 2021). Por otro lado, este elemento no se considera esencial para las plantas, pero podría ser considerado un elemento benéfico debido a que en bajas concentraciones él. Se aumenta el rendimiento, el contenido antioxidante y su concentración en la parte comestible (Preciado-Rangel et al., 2021).

Una estrategia para aumentar el contenido de Se en los alimentos de origen vegetal es mediante la biofortificación que consiste en potenciar la bioactividad y el contenido de Se en las partes comestibles de las plantas Gaucin-Delgado et al. (2020). La fertilización foliar es la manera más practica de para incorporar el Se a la cadena alimenticia (Lyons, 2018). Por otro lado, el chile jalapeño (Capsicum annuиm L.), una de las plantas más cultivadas en el mundo, debido a su importancia en la nutrición humana como en la industria farmacéutica (EspinosaPalomeque et al., 2020), los frutos de son una fuente de vitaminas. (A, E y C), carotenoides, capsaicinoides y compuestos fenólicos con propiedades antioxidantes para la dieta humana (Natividad-Torres et al., 2021).

La aplicación de micronutrientes a través de la biofortificación de cultivos es una herramienta muy útil no solo para incrementar la cantidad de los micronutrientes esenciales sino, además mejorar la biosíntesis de compuestos bioactivos (Gaucin-Delgado et al., 2020). El objetivo de este trabajo fue determinar el efecto de la biofortificación foliar con Selenio sobre el rendimiento, la calidad nutracéutica y la capacidad antioxidante del cultivo de chile jalapeño. 


\section{Materiales y métodos}

\section{Material vegetal y condiciones de crecimiento}

La investigación se realizó en un invernadero con un sistema de enfriamiento automático ubicado en las instalaciones de la Universidad Autónoma Antonio Agraria Antonio Narro (UAAAN) en la Ciudad de Torreón, Coahuila, México ( $25^{\circ} 33^{\prime} 26^{\prime \prime}$ latitud norte, $103^{\circ} 22^{\prime} 31^{\prime}$ ' longitud oeste, a una altitud de $1230 \mathrm{~m}$ ). El cultivo de estudio fue el chile jalapeño cv. Hijo de Mitla germinó en bandejas de poliestireno con 200 huecos rellenos de turba (Premier ${ }^{\circledR}$, México) como sustrato. Estas bandejas de plantas se cubrieron con plástico negro durante $72 \mathrm{~h}$ y se regaron cada $24 \mathrm{~h}$. El trasplante se realizó a los 45 días de la siembra de la semilla cuando las plantas presentaron una altura promedio de $150 \mathrm{~mm}$, cada planta por maceta. Las macetas eran bolsas negras de polietileno de 500 de espesor y $18 \mathrm{~L}$ de capacidad, rellenas con diferentes proporciones de arena:perlita (80:20 vv). La arena del río se lavó y desinfectó con una solución de hipoclorito de sodio al 5\%. Las bolsas se colocaron en doble hilera a $30 \mathrm{~cm}$ de centro a centro entre bolsas y $1.6 \mathrm{~m}$ entre hileras, para obtener una densidad de plantas de 4.2 plantas $\mathrm{m}^{-2}$.

Los requerimientos de agua del cultivo fueron proporcionados por riego manual para proporcionar tres riegos por día, y cada planta recibió $0.6 \mathrm{~L}$ en cada riego, desde el trasplante hasta el inicio de la floración y de 2.5 a $3.5 \mathrm{~L}$ desde la floración hasta la cosecha. La polinización se realizó con cepillo eléctrico diariamente, desde el inicio de la floración hasta el cuajado. La temperatura mínima y máxima dentro del invernadero fluctuó entre 17.7 y $31.6{ }^{\circ} \mathrm{C}$, respectivamente, mientras que la humedad relativa mínima y máxima osciló entre 30 y $70 \%$.

\section{Tratamientos y diseño experimental}

Se utilizó un diseño experimental completamente aleatorizado, aplicando cinco dosis: 0, 1.5, 3, 4.5 y $6 \mathrm{mg} \mathrm{L}^{-1} \mathrm{de} \mathrm{Na}_{2} \mathrm{SeO}_{3}$ (Sigma Aldrich) (León-Morales et al., 2019). Los tratamientos con $\mathrm{Se}$ fueron aplicados cada 15 días con un total de seis aplicaciones a través de fertilización foliar. Se rego con solución nutritiva (Steiner, 1984), el pH y la conductividad eléctrica se mantuvieron en 5.5 y $2 \mathrm{dS} \mathrm{m}^{-1}$ respectivamente. Se determinó el rendimiento, la calidad nutracéutica del fruto y la acumulación de selenio en frutos. Se utilizaron diez plantas por tratamiento.

\section{Rendimiento del cultivo}

En cada unidad experimental, el rendimiento se estimó considerando el número y el peso de la fruta individual por planta, registrando los rasgos individuales de la fruta como el peso con una balanza (Ohaus $3729^{\circledR}$, México).

\section{Calidad nutracéutica}

Extracto de preparación: para la determinación de la calidad nutracéutica (compuestos fenólicos, flavonoides y capacidad antioxidante), se mezclaron muestras de $2 \mathrm{~g}$ de fruto fresco con $10 \mathrm{ml}$ de etanol en tubos plásticos se cerraron con tapón de rosca. Se uso un agitador tipo 'Stuart' para mantener la mezcla en agitación durante $24 \mathrm{~h}$. Después se centrifugaron los tubos a $3000 \mathrm{rpm}$ durante 5 min (Cardeño, 2007). Los sobrenadantes se extrajeron para su análisis físico-químico. 


\section{Contenido fenólico total}

Se determinó mediante una modificación del método de Folin-Ciocalteau (Singleton, 1999). Se tomaron $50 \mu \mathrm{l}$ de extracto etanólico, se diluyeron en $3 \mathrm{ml}$ de agua $\mathrm{mQ}$, se agregaron $250 \mu \mathrm{l}$ de Folin-Ciocalteau $(1 \mathrm{~N})$, se agitó y se dejó reaccionar durante $3 \mathrm{~min}$. Posteriormente, se agregaron $750 \mu \mathrm{l}$ de Na2CO3 (20\%) y $950 \mu \mathrm{l}$ de agua mQ. La solución se dejó reposar durante 2 h y las muestras se midieron en un espectrofotómetro UV-Vis (CGoldenwall, rango de longitud de onda 340-1 $000 \mathrm{~nm}$ y un ancho de banda espectral: 5 a $760 \mathrm{~nm}$. La solución estándar se preparó con ácido gálico. Los resultados fueron expresados en $\mathrm{mg} 100 \mathrm{~g}^{-1}$ de peso fresco.

\section{Flavonoides totales}

Se determinaron mediante espectrofotometría Lamaison (1990). Se tomaron $250 \mu 1$ de extracto etanólico y se mezcló con $1.25 \mathrm{ml}$ de agua mQ y $75 \mu \mathrm{l}$ de $\mathrm{NaNO}_{2}(5 \%)$, se dejó $5 \mathrm{~min}$ y se agregaron $150 \mu \mathrm{l}$ de $\mathrm{AlCl} 3$ (10\%). Posteriormente, se añadieron $500 \mu \mathrm{l}$ de $\mathrm{NaOH}$ (1 M) y $275 \mu 1$ de agua mQ. Se agitó vigorosamente y las muestras se cuantificaron en un espectrofotómetro UV-Vis (CGoldenwall, rango de longitud de onda 340-1 $000 \mathrm{~nm}$ y ancho de banda espectral: 5 a $510 \mathrm{~nm}$. El estándar se preparó con quercetina disuelta en etanol absoluto $\left(\mathrm{y}=0.0122 \mathrm{x}-0.0067 ; \mathrm{r}^{2}=0.965\right)$. Los resultados se expresaron en $\mathrm{mg} 100 \mathrm{~g}^{-1}$ de peso fresco.

\section{Capacidad antioxidante total}

Se midió por el método DPPH + in vitro (Brand-Williams, 1995). Se preparó una solución de $\mathrm{DPPH}+($ Aldrich $)$ en etanol, a una concentración de $0.025 \mathrm{mg} \mathrm{ml}^{-1}$. Se mezclaron $50 \mu 1$ del extracto etanólico con $1.95 \mu \mathrm{l}$ de solución DPPH +, luego de $30 \mathrm{~min}$ las muestras se cuantificaron en un espectrofotómetro UV-Vis (CGoldenwall, rango de longitud de onda a 340-1 $000 \mathrm{~nm}$ y un ancho de banda espectral: 5 a $517 \mathrm{~nm}$. Los resultados se expresaron en equivalente a $\mu \mathrm{M}$ equiv Trolox $100 \mathrm{~g} \mathrm{~m}^{-1}$ peso fresco.

\section{Capsaicina}

El contenido de capsaicina se midió mediante una adaptación del método propuesto por CisnerosPineda et al. (2007). La absorbancia del extracto filtrado se obtuvo luego en un espectrofotómetro $\mathrm{UV} / \mathrm{V}$ is (Goldenwall, rango de longitud de onda a 340-1000 nm) previamente calibrado con acetonitrilo como blanco a una longitud de onda de $273 \mathrm{~nm}$. El contenido de capsaicina se calculó mediante una curva estándar utilizando capsaicina (Sigma, St. Louis, Missouri, EE. UU.) Como estándar y los resultados se expresan en $\mathrm{mg} \mathrm{g}^{-1}$ en peso fresco de capsaicina. Los análisis se realizaron por triplicado.

\section{Acumulación de selenio en el cultivo}

Las muestras de chile seco se molieron en un mortero de porcelana y se digirieron con ácido nítrico y perclórico (3: 1), utilizando una placa calefactora a $100^{\circ} \mathrm{C}$. La solución se filtró y se hirvió para obtener $100 \mathrm{ml}$ de solución de trabajo con agua desionizada. La concentración de selenio en frutos de tomate se determinó mediante espectrofotometría de absorción atómica (Hsieh y Ganther, 1975) los resultados se expresaron en $\mu \mathrm{g} \mathrm{kg}^{-1}$ de peso seco de frutos. 


\section{Análisis estadístico}

La normalidad y homogeneidad de las varianzas de los datos obtenidos se verificaron mediante las pruebas de Kolmogorov-Smirnov y Bartlett, respectivamente. Posteriormente, se realizó análisis de varianza de clasificación simple y comparación múltiple de medias mediante la prueba de Tukey a una probabilidad del 5\% ( $p \leq 0.05)$, con la ayuda del paquete de análisis estadístico SAS v 9.0 (SAS Institute, 2004).

\section{Resultados y discusión}

\section{Rendimiento}

La adición de Se, no afecto significativamente el rendimiento (Figura 1), debido a que el Se, no se considera elemento esencial para el metabolismo de las plantas, no se esperaría que la suplementación con Se cause cambios en el crecimiento y rendimiento de los cultivos HernándezHernández et al. (2019); Sin embargo, se ha reportado que al aplicar dosis altas de Se reduce el rendimiento de los cultivos, debido a que causa un estrés oxidativo en la planta, lo que puede incrementar la producción de radicales libre al defenderse del efecto toxico provocado por las ERO (Hibaturrahman et al., 2020), al contrario, al utilizar bajas dosis, se incrementa el rendimiento (Rady et al., 2020) y evita la oxidación al regular la captación y redistribución de elementos esenciales en los sistemas antioxidantes o manteniendo el equilibrio iónico y la integridad estructural de la célula (Pannico et al., 2019), permitiéndole a la planta adaptarse metabólicamente como fisiológicamente como respuesta al ataque del estrés oxidativo causado por el incremento de radicales libres bajo condiciones de estrés (Hasanuzzaman et al., 2020).

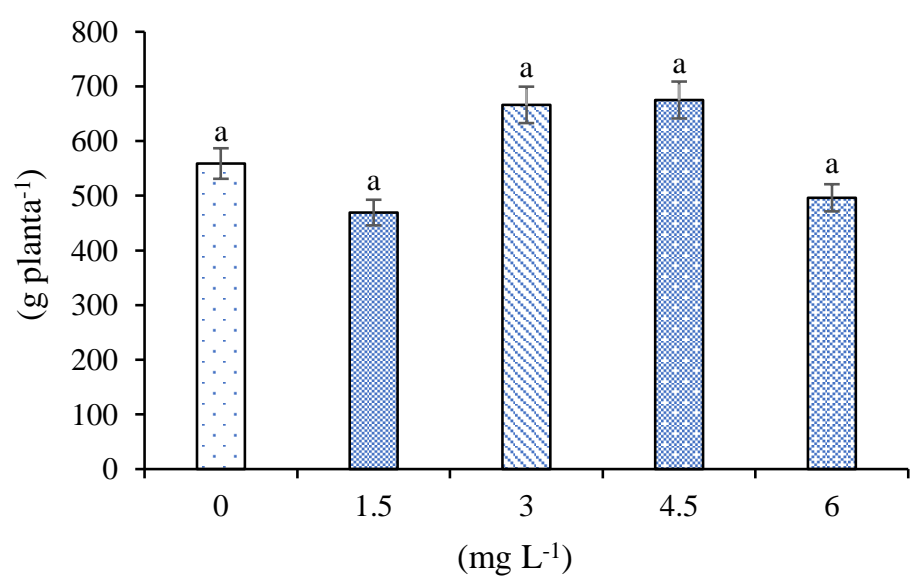

Figura 1. Efecto del Se sobre el rendimiento de frutos de chile. Los datos se muestran como media \pm desviación estándar (DE) $(n=50)$. Columnas con letras diferentes son significativamente diferentes según la prueba de Tukey $(p<0.05)$.

La planta presenta respuesta diferencial al Se dependiendo de la dosis utilizada, tiene un impacto tanto positivo como negativo en las plantas, ya que en concentraciones bajas, el Se acumula como estimulante al promueven el crecimiento, la fisiología de las plantas, aumentan el rendimiento (Gaucin-Delgado et al., 2020) y en dosis altas se ejerce efectos negativos en el crecimiento y la fisiología de la planta (Silva et al., 2020) e incluso muerte celular (Shahid et al., 2018). 


\section{Compuestos bioactivos}

La biosíntesis de compuestos fenólicos, flavonoides y capacidad antioxidante fue influenciada positivamente por la aplicación de Se (Figura 2a-2c), obteniéndose los mayores valores con $6 \mathrm{mg}$ $\mathrm{L}^{-1}$. Diversas investigaciones han demostrado que la aplicación de Se, puede incrementar la producción de compuestos bioactivos (Motesharezadeh et al., 2020). Resultados similares a los del presente estudio fueron obtenidos en Capsicum annuиm L., donde la aplicación de Se incrementó diversos compuestos bioactivos como fenoles, flavonoides y capacidad antioxidante (NatividadTorres et al., 2021).

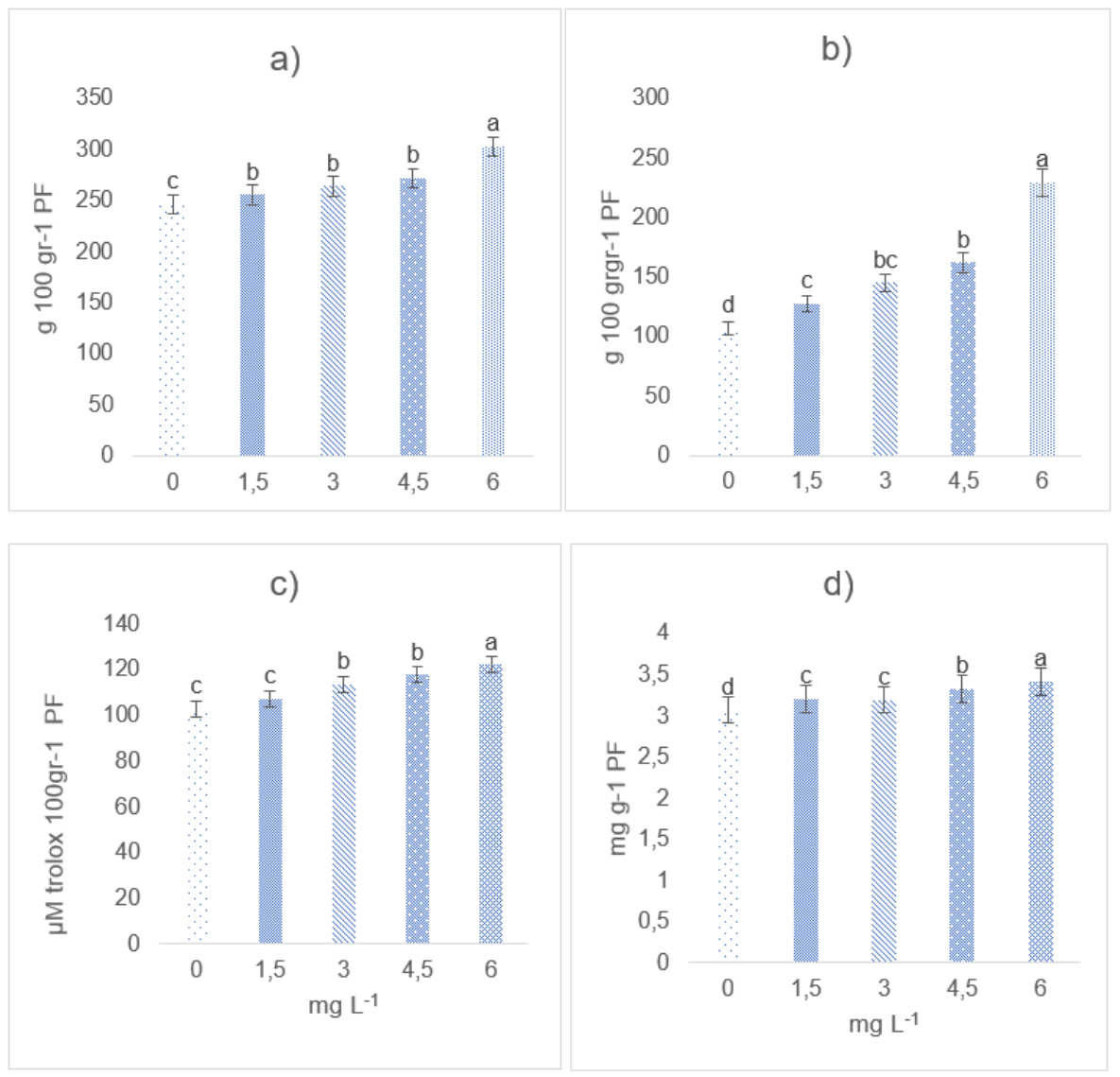

Figura 2. Efecto del Se sobre el contenido de fenólico (a); flavonoides totales (b); capacidad antioxidante (c); y capsaicina (d) en frutos de chile. Los datos se muestran como media \pm desviación estándar (DE) $(n=50)$. Columnas con letras diferentes son significativamente diferentes según la prueba de Tukey $(p<0.05)$.

Esta capacidad bioactiva posiblemente sea atribuida a la acción sinérgica que tiene el Se al poder actuar como un elemento vital al alterar varios procesos fisiológicos y bioquímicos D'Amato et al. (2018), además el Se afecta directamente el sistema de defensa antioxidante al aumenta el potencial de las plantas para sobreponerse a las condiciones de estrés biótico y abiótico (Hachmann et al., 2019). Los resultados obtenidos en nuestro estudio se respaldan por (Hibaturrahman et al., 2020), quienes encontraron que la suplementación en solución nutritiva con $\mathrm{Na}_{2} \mathrm{SeO}_{4}$ eleva la actividad antioxidante protegiendo así a las plantas del estrés oxidativo. 
La producción de alimentos ricos en compuestos bioactivos, influye directamente en las actividades celulares y fisiológicas Sabatino et al. (2019), obteniendo, tras su ingesta, un aporte beneficioso para la salud humana (Shahid et al., 2018) por sus diversas características, de protección en enfermedades de tipo coronario (Groth et al., 2020) y tras evitar el envejecimiento celular (Hernández-Hernández et al., 2019), además de ser usado como una alternativa para la prevención del cáncer (Vinceti et al., 2018).

\section{Capsaicina}

La capsaicina es una oleorresina, componente activo de los pimientos picantes (Friedman et al. (2019). La capsaicina fue afectada positivamente por las dosis de Se evaluadas (Figura 2d). La concentración de capsaicina, estuvo en concordancia con las dosis de Se empleadas, el aumento en la pungencia del chile mejora su calidad, ya que esta característica es apreciada por los consumidores (Uarrota et al., 2021). Los resultados indican una respuesta al contenido de capsaicina en los frutos de chile jalapeño al Se, aunque el mecanismo de acción en este proceso no se ha definido. La biosíntesis está influenciada por las interacciones genotipo-ambiente en frutos de Capsicum (Naves et al., 2019), fenilpropanoideds (Aza-González et al., 2011) y los transportadores ABC, las subfamilias ABCC y ABCG, que pueden estar desempeñando papeles en el transporte de metabolitos secundarios como la capsaicina y dihidrocapsaicina a las vacuolas de placenta, afectando su contenido en frutos (Lopez-Ortiz et al., 2019).

Probablemente a la interacción del Se en la planta al ocasionarle efectos estresantes Avery y Hoffmann (2018) que pudieran estar relacionados con un aumento la actividad de PAL y capsaicina sintasa aumentando la acumulación de capsaicinoides en el chile (Ulloa et al., 2021), al regular negativamente la actividad de peroxidasa a niveles apropiados (Hernández-Pérez et al., 2020).

Aunque la biosíntesis es un rasgo genético controlado, el medio ambiente también juega un papel importante dependiendo del genotipo Scossa et al. (2019) además, esta característica también está regulada desde el punto de vista del desarrollo y del medio ambiente Naves et al. (2019). Por otro lado, la capsaicina proporciona la sensación oral picante en la mayoría de los chiles (Scossa et al., 2019); sin embargo, sus propiedades biológicas más importantes es su capacidad para actuar como antioxidantes para reducir el estrés oxidativo que conduce a la prevención de varias enfermedades degenerativas (Friedman et al., 2019).

\section{Contenido de Selenio en el cultivo}

La adición de $6 \mathrm{mg} \mathrm{L}^{-1}$ aumento el contenido de Se en los frutos de chile, existe una correlación positiva entre el Selenio en frutos y su disponibilidad $\left(r^{2}=0.98\right)$ (Figura 3). La absorción del Se dependen de la edad de la planta, especie vegetal y la forma química del elemento que se aplica, su concentración y el método de aplicación (Yin et al., 2019). Los vegetales acumulan mayores cantidades de Se (Dai et al., 2019).

Estudios previos muestran que la biofortificación aumenta significativamente la cantidad de elementos esenciales en la parte comestible de las plantas Silva et al. (2020), la cual puede aumentar en los cultivos biofortificados con Se hasta $30 \%$ con respecto a los no tratados (Zhu et al., 2017). El Se en las plantas se metaboliza junto con el azufre dentro de los tejidos vegetales, transformándose en selenoproteinas que permiten almacenarse como Se-Met (Zhang et al., 2019), acelerando el transporte, la acumulación, la volatilización y la tolerancia al Se (Raina et al., 2020). 


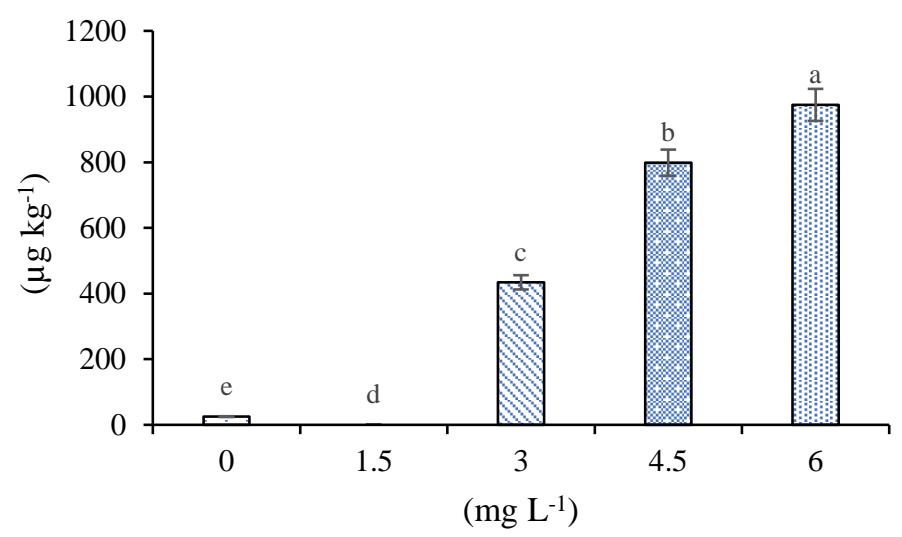

Figura 3. Concentración de Se en frutos de chile. Los datos se muestran como media \pm desviación estándar (DE) $(\mathbf{n}=\mathbf{5 0})$. Las columnas con letras diferentes son significativamente diferentes según la prueba de Tukey $(p<0.05)$.

Actuando como un potente antioxidante, al permitir menores niveles de peroxidación lipídica y mayor actividad de enzimas antioxidantes, así como una mejor resistencia al estrés oxidativo (Skrypnik et al. (2019). Según la guía alimentaria para los estadounidenses, se debe obtener la mayoría de los nutrientes requeridos con la ingesta de alimentos (Padilla-Samaniego et al., 2020). En este sentido el consumo de $0.74 \mathrm{~g}$ de chile jalapeño biofortificadado con selenio, suple las necesidades diarias de este oligoelemento que es de $55 \mathrm{mg}$ por día en adulto (Chomchan et al., 2017; León-Morales et al., 2019).

\section{Conclusiones}

La biofortificación agronómica con selenio mejora la calidad nutracéutica y la concentración de Se, sin afectar el rendimiento del cultivo de chile jalapeño, la utilización de Se es una alternativa para obtener alimentos funcionales y aumentar la acumulación del oligoelemento en los frutos de chile jalapeño, con la posibilidad de proteger la salud humana con su consumo.

\section{Literatura citada}

Avery, J. C. and Hoffmann, P. R. 2018. Selenium, selenoproteins, and immunity. Nutrients. 10(9):1203-1223.

Aza-González, C.; Núñez-Palenius, H. G. and Ochoa-Alejo, N. 2011. Molecular biology of capsaicinoid biosynthesis in chili pepper (Capsicum spp.). Plant Cell Reports. 30(5):695-706.

Błażewicz, A.; Szymańska, I.; Dolliver, W.; Suchocki, P.; Turło, J.; Makarewicz, A. and Skórzyńska-Dziduszko, K. 2020. Are obese patients with autism spectrum disorder more likely to be selenium deficient?. Research findings on pre-and post-pubertal children. Nutrients. 12(11):3581.

Brand-Williams, W.; Cuvelier, M. E. and Berset, C. 1995. Use of a free radical method to evaluate antioxidant activity. LWT-Food Science Technology. 28(1):25-30

Cardeño, Á.V.; Molina, M. C.; Miranda, I.; García, G. T.; Morales, J. M. and Stashenko, E. E. 2007. Actividad antioxidante y contenido total de fenoles de los extractos etanólicos de Salvia aratocensis, Salvia Sochensis, Bidens reptons y Montanoa ovalifolia. Scientia et Technica.13(33):205-207. 
Chomchan, R.; Siripongvutikorn, S. and Puttarak, P. J. 2017. Selenium bio-fortification: an alternative to improve phytochemicals and bioactivities of plant foods. Functional Foods in Health Disease. 7(3):263-279.

D’Amato, R.; Fontanella, M. C.; Falcinelli, B.; Beone, G. M.; Bravi, E.; Marconi, O.; Benincasa, P. and Businelli, D. J. 2018. Selenium biofortification in rice (Oryza sativa L.) sprouting: effects on Se yield and nutritional traits with focus on phenolic acid profile. J. of Agric. Food Chem. 66(16):4082-4090.

Dai, H.; Wei, S.; Skuza, L. and Jia, G. 2019. Selenium spiked in soil promoted zinc accumulation of Chinese cabbage and improved its antioxidant system and lipid peroxidation. Ecotoxicol. Environ. Safety. 180(19):179-184.

Espinosa-Palomeque, B.; Cano-Ríos, P.; Salas-Pérez, L.; González-Rodríguez, G.; ReyesGonzález, A.; Ayala-Garay, A. V. and Preciado-Rangel, P. J. 2020. Vermicompost on the production and nutraceutical quality of jalapeño pepper fruits (Capsicum annuum L.). Terra Latinoam. 38(4):795-803.

Friedman, J. R.; Richbart, S. D.; Merritt, J. C.; Brown, K. C.; Denning, K. L.; Tirona, M. T.; Valentovic, M. A.; Miles, S. L. and Dasgupta, P. 2019. Capsaicinoids: multiple effects on angiogenesis, invasion and metastasis in human cancers. Biomedicine Pharmacotherapy. 118(109317):2-9.

Gaucin-Delgado, J. M.; Hernandez-Montiel, L. G.; Sanchez-Chavez, E.; Ortega-Ortiz, H.; FortisHernandez, M.; Reyes-Pérez, J. J. and Preciado-Rangel, P. 2020. Agronomic biofortification with selenium improves the yield and nutraceutical quality in tomato under soilless conditions. Notulae Botanicae Horti Agrobotanici Cluj-Napoca. 48(3):1221-1232.

Górska, S.; Maksymiuk, A. and Turło, J. 2021. Selenium-containing polysaccharides structural diversity, biosynthesis, chemical modifications and biological activity. Appl. Sci. 11(8):3717.

Groth, S.; Budke, C.; Neugart, S.; Ackermann, S.; Kappenstein, F. S.; Daum, D. and Rohn, S. 2020. Influence of a selenium biofortification on antioxidant properties and phenolic compounds of apples (Malus domestica). Antioxidants. 9(2):187-209.

Hachmann, T. L.; Rezende, R.; Matumoto-Pintro, P. T.; Saath, R.; Anjo, F. A. and Menezes, C. S. L. 2019. Yield, antioxidant activity and shelf-life of cauliflower inflorescences under drought stress and foliar spraying of selenium. Ciência e Agrotecnologia. 43 p.

Hasanuzzaman, M.; Bhuyan, M. B.; Raza, A.; Hawrylak-Nowak, B.; Matraszek-Gawron, R.; Al Mahmud, J.; Nahar, K. and Fujita, M. 2020. Selenium in plants: boon or bane? Environ. Exp. Bot.178(10):104170.

Hernández-Hernández, M.; León-Morales, J.; López-Bibiano, Y.; Saldaña-Sánchez, W. D. and García-Morales, S. 2019. Efecto comparativo del selenito y selenato en el crecimiento y contenido de pigmentos fotosintéticos en plantas de pimiento (Capsicum annuиm L.) Biotecnología y Sustentabilidad. 3(2):12-12.

Hernández-Pérez, T.; Gómez-García, M. D. R.; Valverde, M. E. and Paredes-López, O. J. 2020. Capsicum annuum (hot pepper): An ancient Latin-American crop with outstanding bioactive compounds and nutraceutical potential. A review. Comprehensive Reviews in Food Science Food Safety. 19(6):2972-2993.

Hibaturrahman, S. N.; Koyama, H.; Kameo, S.; Waspodo, P.; Wardana, A. A. and Surono, I. S. 2020. Effect of cocoyam modified starch (Xanthosoma sagittifolium), beetroot juice, cocoyam modified starch adsorbing beetroot on plasma selenium and glutathione peroxidase of pre-diabetic rat. IOP Conference Series: Earth and Environmental Science. IOP Publishing. 426(1):012184. 
Hsieh, H. S. and Ganther, H. E. 1975. Acid-volatile selenium formation catalyzed by glutathione reductase. Biochemistry. 14(8):1632-1636.

Khurana, A.; Tekula, S.; Saifi, M. A.; Venkatesh, P. and Godugu, C. 2019. Therapeutic applications of selenium nanoparticles. Biomedicine Pharmacotherapy. 111(12):802-812.

Lamaison, J. and Carnet, A. 1990. The levels of the main flavonoids completing of the flower of Craaegeus monogyna Jawq and Crataegeus laevigata (poiret) as a function of the vegetation. Pharm Acta Helv. 65(1):315-320.

León-Morales, J.; Panamá-Raymundo, W.; Langarica-Velázquez, E. and García-Morales, S. 2019. Selenio y vanadio en la germinación y el crecimiento de plántulas de chile (Capsicum annuum L.) y rábano (Raphanus sativus). Rev. Bio Ciencias. 6(10): e425-e441.

Lopez-Ortiz, C.; Dutta, S. K.; Natarajan, P.; Peña-Garcia, Y.; Abburi, V.; Saminathan, T.; Nimmakayala, P. and Reddy, U. K. 2019. Genome-wide identification and gene expression pattern of ABC transporter gene family in Capsicum spp. PloS One. 14(4): e0215901e0215924.

López, E. A. T.; Sandoval-Rangel, A.; Mendoza, A. B.; Ortiz, H. O.; Pliego, G. C. and de la Fuente, M. C. 2021. Nanopartículas de selenio absorbidas en hidrogeles de quitosán-polivinil alcohol en la producción de pepino injertado. Rev. Mex. Cienc. Agríc. 6(26):159-169.

Lyons, G. 2018. Biofortification of cereals with foliar selenium and iodine could reduce hypothyroidism. Front. Plant Sci. 9(730):2-8.

Motesharezadeh, B.; Ghorbani, S. and Alikhani, H. A. 2020. The effect of selenium biofortification in alfalfa (Medicago sativa). J. Plant Nutr. 43(2):240-250.

Natividad-Torres, E. A.; Guevara-Aguilar, A.; Sánchez, E.; Sida-Arreola, J. P.; Muñoz-Márquez, E. and Chávez-Mendoza, C. J. 2021. Effect of the processing on the antioxidant capacity and bioactive compounds content of jalapeno pepper for chipotle and commercial sauces. Acta Agríc. Pec. 7(1):E00710007-E00710009.

Naves, E. R.; de Ávila Silva, L.; Sulpice, R.; Araújo, W. L.; Nunes-Nesi, A.; Peres, L. E. and Zsögön, A. 2019. Capsaicinoids: pungency beyond Capsicum. Trends Plant Sci. 24(2):109-120.

Padilla-Samaniego, M. V.; Naranjo-Rodriguez, C. E.; Ramírez-Anormaliza, R. I.; Lozada-Meza, M. L.; Solís-Manzano, A. M. and Calderón-Vallejo, C. V. 2020. Tamaño y porciones del consumo de alimentos de la población: disponibilidad de información actualizada. Rev. Eugenio Espejo.14(2):30-50.

Pannico, A.; El-Nakhel, C.; Kyriacou, M. C.; Giordano, M.; Stazi, S. R.; De Pascale, S. and Rouphael, Y. 2019. Combating micronutrient deficiency and enhancing food functional quality through selenium fortification of select lettuce genotypes grown in a closed soilless system. Front. Plant Sci. 10(19):1495-1534.

Preciado-Rangel, P.; Hernández-Montiel, L. G.; Valdez-Cepeda, R. D.; Cruz-Lázaro, E. D. L.; Lara-Capistrán, L.; Morales-Morales, B. and Gaucin-Delgado, J. M. 2021. Biofortification with selenium increases bioactive compounds and antioxidant capacity in tomato fruits. Terra Latinoam. 39(e979):1-10.

Rady, M. M.; Belal, H. E.; Gadallah, F. M. and Semida, W. M. 2020. Selenium application in two methods promotes drought tolerance in Solanum lycopersicum plant by inducing the antioxidant defense system. Scientia Hortic. 9(34):113-128.

Raina, M.; Sharma, A.; Nazir, M.; Kumari, P.; Rustagi, A.; Hami, A.; Bhau, B. S.; Zargar, S. M. and Kumar, D. 2020. Exploring the new dimensions of selenium research to understand the underlying mechanism of its uptake, translocation, and accumulation. Physiol. Plantarum. 171(4):882-895. 
Sabatino, L.; Ntatsi, G.; Iapichino, G.; D’Anna, F. and De Pasquale, C. 2019. Effect of selenium enrichment and type of application on yield, functional quality and mineral composition of curly endive grown in a hydroponic system. Agronomy. 9(4):207-222.

Sariñana-Navarrete, M. D. Á.; Hernández-Montiel, L. G.; Sánchez-Chavez, E.; Reyes-Perez, J. J.; Murillo-Amador, B.; Reyes-González, A. and Preciado-Rangel, P. 2021. Foliar fertilization of sodium selenite and its effects on yield and nutraceutical quality in grapevine. Rev. Fac. Agron. 38(4):806-824.

Schiavon, M.; Nardi, S.; Dalla, V. F. and Ertani, A. 2020. Selenium biofortification in the $21^{\text {st }}$ century: status and challenges for healthy human nutrition. Plant Soil. 453(1):1-26.

Scossa, F.; Roda, F.; Tohge, T.; Georgiev, M. I. and Fernie, A. R. 2019. The hot and the colorful: understanding the metabolism, genetics and evolution of consumer preferred metabolic traits in pepper and related species. Critical Rev. Plant Sci. 38(6):339-381.

Shahid, M.; Niazi, N. K.; Khalid, S.; Murtaza, B.; Bibi, I. and Rashid, M. I. 2018. A critical review of selenium biogeochemical behavior in soil-plant system with an inference to human health. Environ. Pollut. 234(17):915-934.

Singleton, V. L.; Orthofer, R. and Lamuela-Raventós, R. M. 1999. Analysis of total phenols and other oxidation substrates and antioxidants by means of folin-ciocalteu reagent. Methods in Enzymology. 299(14):152-178.

Silva, D. F.; Cipriano, P. E.; de Souza, R. R.; Júnior, M. S.; Faquin, V.; de Souza Silva, M. L. and Guilherme, L. R. G. 2020. Biofortification with selenium and implications in the absorption of macronutrients in Raphanus sativus L. J. Food Comp. Analysis. 86(18):103382-103409.

Skrypnik, L.; Kurkova, T. and Chupakhina, G. 2019. Accumulation of selenium in rye plants (Secale cereale L.) at different stages of development and grain quality due to selenate soil supplementation. Appl. Ecol. Environ. Res. 17(2):2385-2421.

Sonkusre, P. 2020. Specificity of biogenic selenium nanoparticles for prostate cancer therapy with reduced risk of toxicity: an in vitro and in vivo study. Front. Oncol. 9(1):1541-1559.

Steiner, A. A. 1984. The universal nutrient solution. International Congress on Soilless ISOSC. $1: 25$

Uarrota, V. G.; Maraschin, M.; de Bairros, Â. D. F. M. and Pedreschi, R. 2021. Factors affecting the capsaicinoid profile of hot peppers and biological activity of their non-pungent analogs (Capsinoids) present in sweet peppers. Crit. Rev. Food Sci. Nutr. 61(4):649-665.

Ulloa, J.; Muñoz, J. S. C.; Barrios, A. V. F.; Van Uden, E. and Tafur, S. 2021. Importancia y beneficios del consumo de huevo de gallina enriquecido con selenio: revisión narrativa. Rev. Nutrición Clínica y Metabolismo. 4(3):124-129.

Vinceti, M.; Filippini, T.; Del Giovane, C.; Dennert, G.; Zwahlen, M.; Brinkman, M.; Zeegers, M. P.; Horneber, M.; D’Amico, R. and Crespi, C. M. 2018. Selenium for preventing cancer. Cochrane Database of Systematic Reviews. 1(1):1465-1858.

Yin, H.; Qi, Z.; Li, M.; Ahammed, G. J.; Chu, X. and Zhou, J. J. 2019. Selenium forms and methods of application differentially modulate plant growth, photosynthesis, stress tolerance, selenium content and speciation in Oryza sativa L. Ecotoxicol. Environ. Safety. 169(19):911-917.

Zhang, L.; Hu, B.; Deng, K.; Gao, X.; Sun, G.; Zhang, Z.; Li, P.; Wang, W.; Li, H. and Zhang, Z. J. 2019. NRT1. 1B improves selenium concentrations in rice grains by facilitating selenomethinone translocation. Plant Biotechnol. J. 17(16): 1058-1068.

Zhu, Z.; Chen, Y.; Shi, G. and Zhang, X. 2017. Selenium delays tomato fruit ripening by inhibiting ethylene biosynthesis and enhancing the antioxidant defense system. Food Chem. 219(1):179-184. 